\title{
A SIMULATOR for ROAD CONSTRUCTION EQUIPMENT
}

\author{
David BÉTAILLE, François PEYRET \\ LABORATOIRE CENTRAL DES PONTS ET CHAUSSÉES - NANTES - FRANCE \\ B.P. 19 - 44340 - BOUGUENAIS - FRANCE \\ Tel : + 33.240.84.56.23/+33.240.84.59.40 \\ E-mail : David.Betaille@lcpc.fr / Francois.Peyret@lcpc.fr
}

\begin{abstract}
The current practices on the road construction worksites have begun to change rapidly and fundamentally since the arrival on the market of new real-time positioning systems.

These systems mean it is now possible to overcome the main weakness existing on construction sites today, e.g.. the huge gap between the design phases which are highly computerized and the work site itself where all numerical data is reduced to wooden grade stakes.

New fields of research and development are now open for those who are in charge of accompanying this evolution: the localization techniques of course, but also the automatic control and guidance, the data transfer between all the different interactors and, from a general point of view, the real-time management of the work site.

To support its activity in this field, the Laboratoire Central des Ponts et Chaussées, has developed a simulation software tool, based on the MATLABSIMULINK environment, which has already proved its efficiency through several research projects.
\end{abstract}

\section{Introduction}

At the present moment, people of the civil engineering world are living a kind of small revolution about their current practices on the job sites, due to the recent arrival on the market of real-time positioning systems.

Actually, a positioning system offers a unique link between the work site phase and the design phase.

Compared to traditional techniques, a new methodology of managing and carrying out the work can be realized through such a link.

Figure 1 shows what the future methodology could be, using positioning systems integrated with adapted software, and fully using the "project" numeric data received from the Computer Aided Design (CAD) [1].

It can be seen that the $\mathrm{CAD}$ software becomes more than a simple design software, but also an interactive geometric database in which the two models (theoretical model of the project and the actual model of the work asbuilt) co-exist.

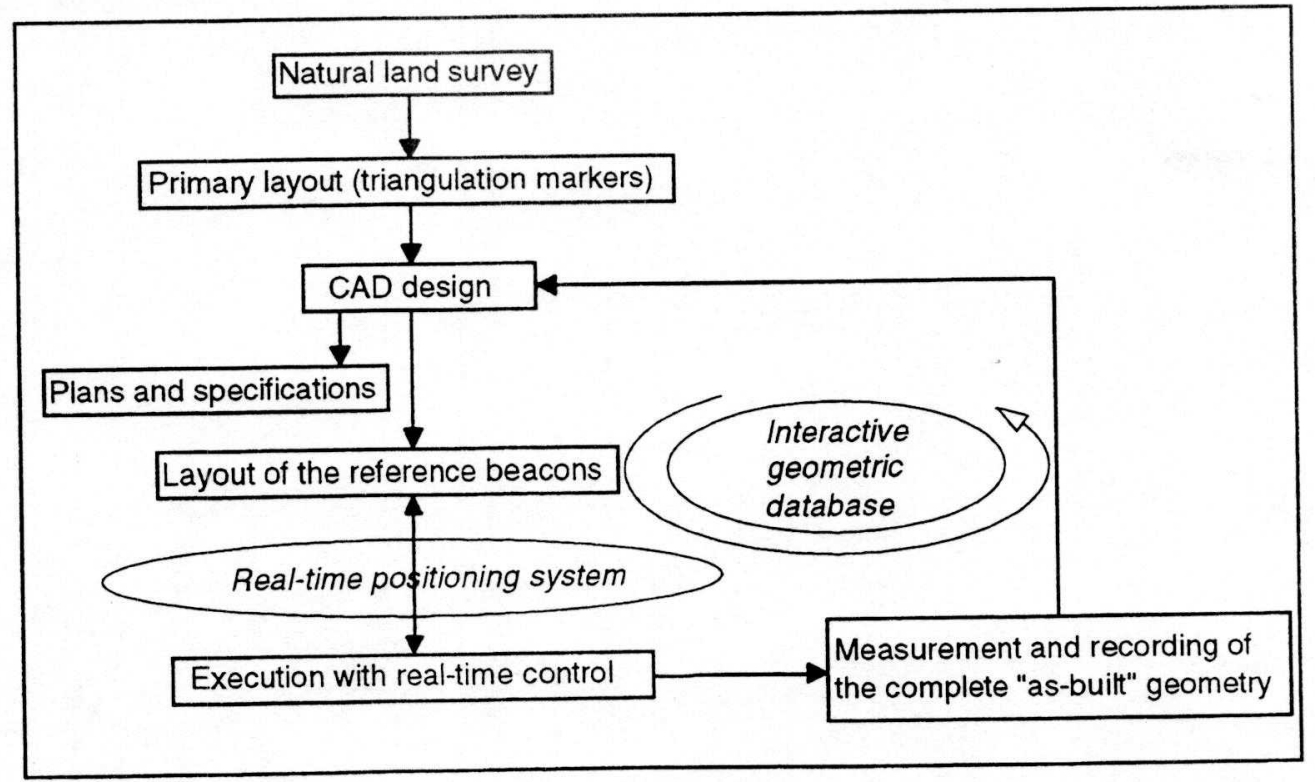

Figure 1 : future methodology using real-time positioning 
The plans are only used as work and dialogue aids and no longer as links in the chain of data transmission.

It is also to be noted that the design-executioncontrol phases become closely linked together (in a loop) and share the same unique data located in the database, reducing the risks of transmission errors to almost zero.

In conclusion, we may say that the technological addition of real-time positioning is opening the door to a new concept which could be termed «computer integrated road construction » [2].

The LCPC (Road Construction Methods and Equipment division) has been working on this new concept from different ponts of view.

From the methodological point of view, new data transfers have to be envisaged, implying new modelization of the road characteristics.

From the technical point of view, some major new issues have to be addressed : the positioning of course, but also the automatic control of the equipment

As a support for these research axes, we have decided, some 3 years ago, to build a simulation software. This tool would allow us to carry out our studies and to assess them through a computer simulation phase, before the full-scale experiments, which are still quite heavy to carry out.

\section{Requirements specifications for the simulator}

SimuCIRC is a software which provides a simulation of a computer integrated road construction application, using initially a paver as an example, but also applicable to other equipment.
A listing of the different potential interactors and of their respective needs leads to the specifications of its main functions.

These interactors are users like :

- road construction companies,

- industrial integration firms,

- site robotics research laboratories.

Different interactors from the environment have to be contemplated too:

- road CAD,

- equipment (machine),

- Iocalisation system,

- operator.

Users...

Companies are looking forward to improving both quality and productivity with computer integrated road construction, and the simulator is showing what seems possible to do. For its credibility, the software should use projects from current road CAD and models of equipment and localisation the most realistic as possible.

Industrial integration firms are interested in applying the analysis performed in a simulation software. This is partly available in future development on equipment. There is also an important new market in connection with localisation systems and integration.

Additionally, the software should provide a most satisfactory research environment in order to study models of equipment, control and localisation systems. This is why not only pavers are considered here, as an example among the existing machines, but also the most important diversity of equipment.

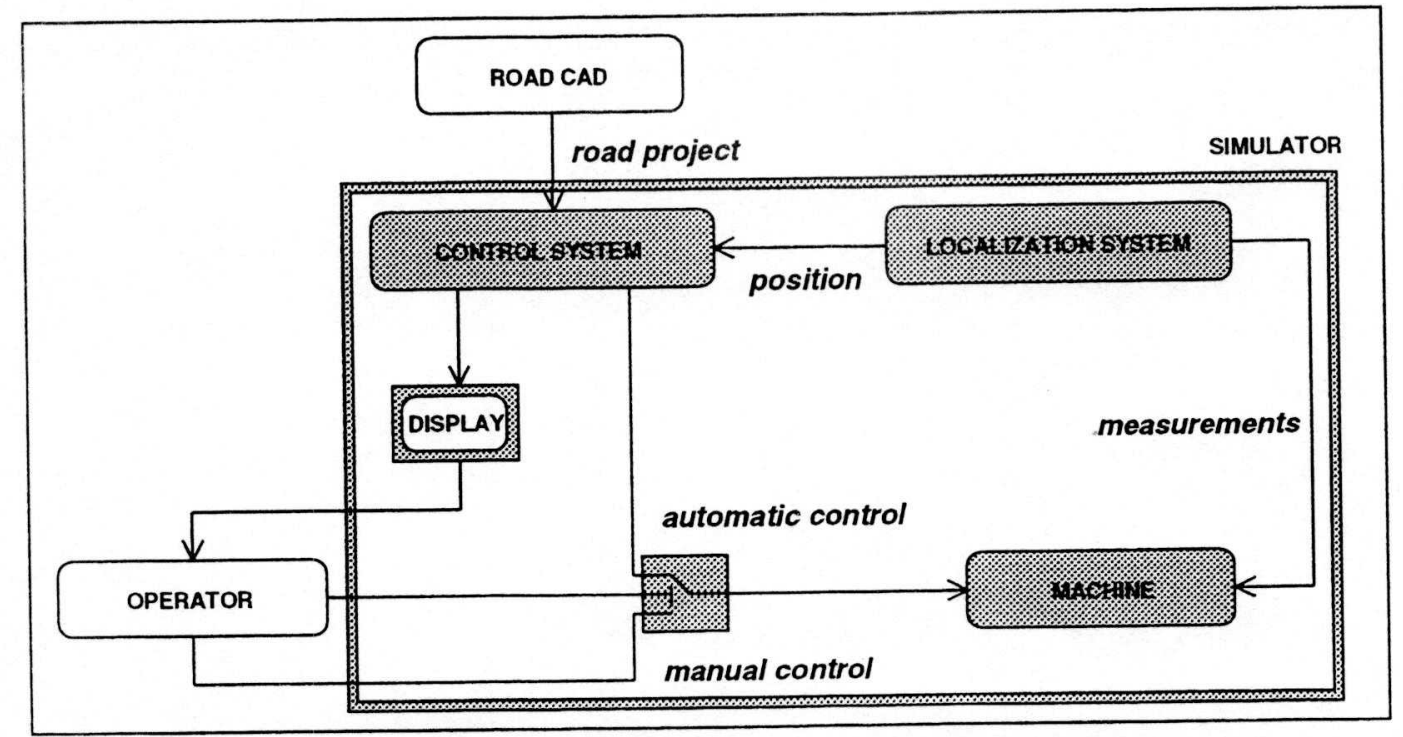

Figure $2:$ an application and its environment, in reality and in simulation

\section{Environment...}

The simulator should be interfaced with several current road CAD systems, in order to validate a protocol for data transfer in an application of computer integrated road construction. 
Models of construction equipment have to be integrated in this software. It should provide the facility necessary to integrate models with different levels of achievement, and for different machines. Kinematic models are at least necessary. Dynamic ones could be useful in a second step.

Besides, models concern also the localisation. They of course must deliver localisation data (« external models »). The most sophisticated of them could compute the data processing itself with its own measurements (« internal models »).

Finally, the simulator should animate a graphical user's interface to let the operator control the simulated models and set the value of manual commands or switch to an automatic command.

Figure 2 represents a real application and its environment. The envelope of the simulator is shown: notice that the $\mathrm{CAD}$ and the operator are not simulated.

\section{Principle of the simulation}

The principle of the simulation relies on a systematic decomposition of a machine into two operational parts : the tractor and the tool or working unit [3].

The simulator makes the tractor move on the support surface, and it makes the tool move with respect to the tractor too. The commands are generated by a control system or by an operator.

While moving, localisation data can be computed and delivered either to the operator or to the control system.

Localisation data can be used then, to evaluate commands in order to cancel deviations of both tractor and working unit compared to the target surface or (specified surface). below.

Support surface and target surface are detailed

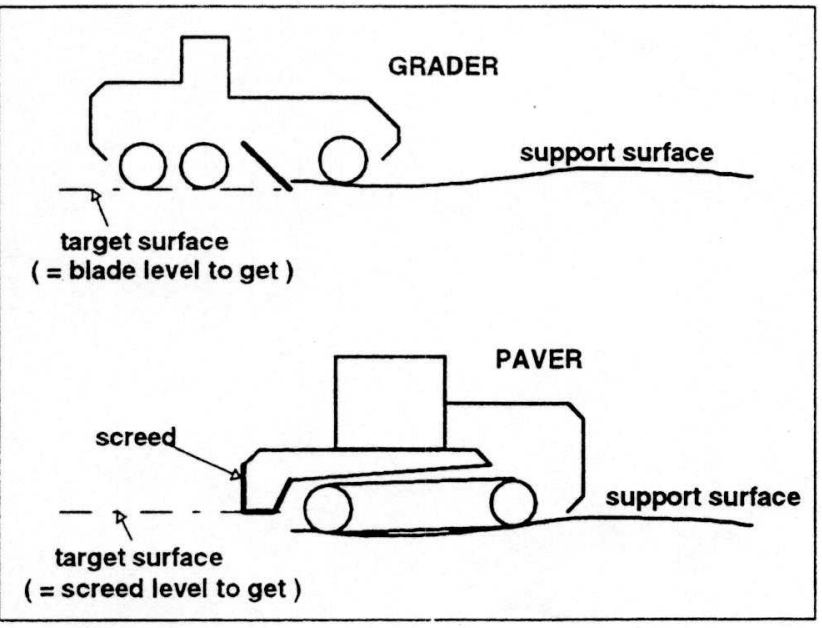

Figure 3 : support surface and target surface

\section{Standard analysis of the simulator}

Let us analyse the simulation from the automatic control theory point of view. The standard control analysis allows the designer to organise the different input and output of a system.

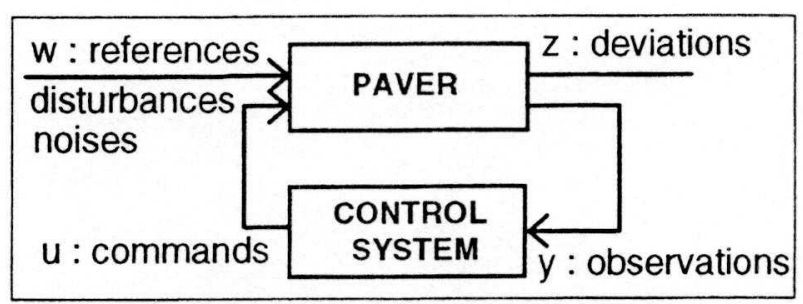

Figure 4 : the standard analysis of the paver simulation

Concerning a floating screed road paver, inputs are :

- references :

- axis of the road,

- profile of the target surface,

- perturbations :

- profile of the support surface,

- variation of the paving process parameters,

- noises :

- noises on simulated localisation data,

deviations are :

- lateral deviation of the tractor with respect to the axis,

- yaw deviation with respect to the direction,

- relative position of the floating screed and the target surface, particularly for altitude,

observations are simulated localisation data, obtained from tractor and tool movements computation :

- 3D coordinates of the extremities of the screed

(e.g. GPS antennas simulation),

- encoder pulses for instance too,

commands are :

- steering angle,

- left actuator length,

- right actuator length.

\section{Hardware and software environment of the simulator}

Every point detailed before is computed and solved step by step during the simulation according to a sample time and integration method.

Therefore, the choice of MATLAB-SIMULINK appears natural for such a simulator. SIMULINK is a simulation toolbox of MATLAB. It allows the designer to edit with blocks and links systems conform with models in automatics, and solve the temporal differential equations the systems contain. Of course, it is necessary to design models before. If standard blocks and links are not enough for a given system, the user can develop 
S-functions whose input and output protocols agree with the calling system.

SIMULINK has connections with libraries of the signal processing toolbox of MATLAB which can be useful to noise measurements.

Besides, MATLAB is convenient to develop a graphical operator's interface and display results after a simulation session. A graphical animation can be easily developed and piloted by a SIMULINK system. Thus, animation lets the operator control and command the calling system throughout the screen.

MATLAB-SIMULINK is compatible with numerous host systems, whatever they are, PC, MAC or UNIX. The currently presented simulation runs on PC Pentium but it automatically fits the host system and the screen size. A large screen (19') is confortable to see simultaneously the main menu of the simulator, the running SIMULINK system, and the animation.

\section{Site modelling in the simulator}

The link with current road CAD tools is a major function of the simulator. For the moment, only one $\mathrm{CAD}$ format is compatible: MICROPISTE. Upgrades for other CAD systems (MOSS, MACAO...) are envisaged and the simulator is designed to be so upgraded.

The simulator explores the axis projection and profiles. It looks for a standard cross-section, in order to permit the operator to choose parameters for the machine, for example: the width of the paver floating screed. The simulation is then only possible on standard cross-section parts of the complete project. The available standard cross-sections are presented on figure 5 .
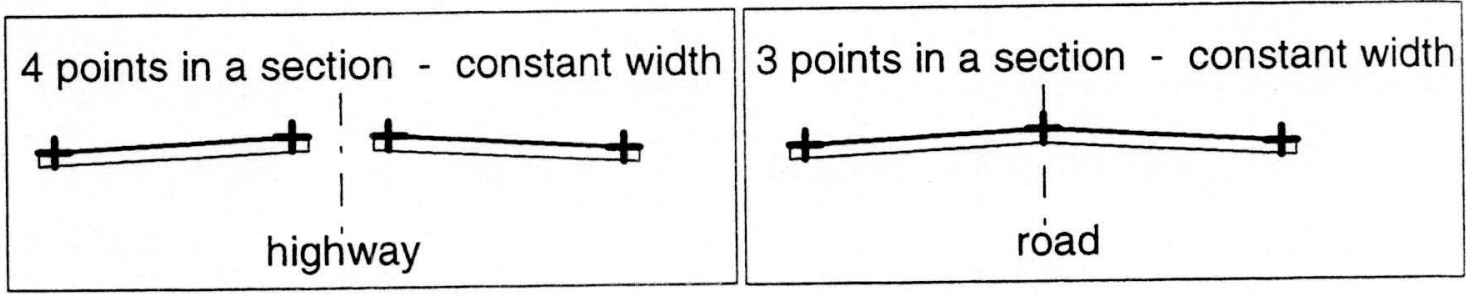

Figure 5 : standard cross-sections

The roads projects when designed in $\mathrm{CAD}$ do not contain the description of numeric data for the different layers. Before a session of simulation, one must choose, for a given project, which layers will be built, with values of thickness, differences of thickness from the shoulders to the median strip, and the extra width in the shoulders and the median strip.

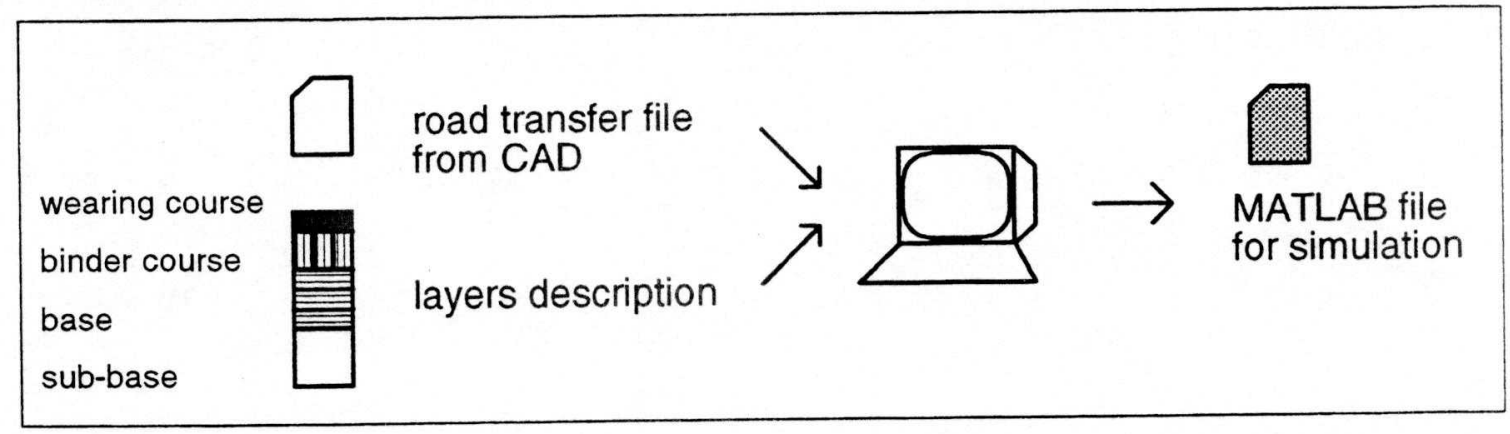

Figure 6 : how CAD road projects are used and completed

Moreover, it is interesting to introduce the tolerance level needed for the layers. The tolerance level concerns the thickness, the gradient, the cross-fall and the extra width. The tolerance level is introduced in order to model and compute a support surface for the movement of the machine throughout the project. It is important that the support surface should not be perfect of course, but with defects introduced in it. The defects should refer to the tolerance level. Notice that the tolerance level is also introduced to switch alarms to eventually warn the operator of any problem.
About defects of the support surface, let us make the assumption that base paving is simulated. The target surface is obtained from the project by the description of the upper layers to the base itself. The support surface is the sub-base, which have been described like the other layers. And the tolerance level of the sub-base can give an idea of the defects one can imagine to move on with the paver. In the simulator, these defects are computed automatically, according to default values (tolerance level multiplied by 2 ) or values chosen by the operator 
Defects can be envisaged in term of eveness. To design this part of the simulator, researches from the LCPC were very useful, particularly those concerning the Analyseur de Profil en Long Numérique (virtual roughness analyser «APLN »).

Here are the successive steps of the process developed to obtain the support surface :

- the axis is cut in stretches of random lengths

- defaults lengths limits are 0.7 and $44.8 \mathrm{~m}$, significant for the APLN, or they are chosen by the operator

- a triangle signal is added vertically to the edge of the road, near the median strip

- its lengths are the same as the lengths of the stretches

- its amplitude is limited by the thickness tolerance level multiplied by 2 or by a value chosen by the operator

- a triangle signal is added vertically to the edge of the road, opposite the median strip

exactly the same method as near the median strip, but the gradient and cross-fall variations due to both triangle signals must not exceed values referenced to the tolerance level

- both triangle signals are smoothed by parabolic links.

The support surface so computed will excite the control system of the floating screed (tool) level: here two actuators. The axis of the project, generally not always a straight line, will excite the control system of the tractor steering.
Of course, the software contains functions to interpolate exactly the position, the axis direction, the gradient and the cross-fall in any point knowing its curvilinear abscissa along the project. These functions are used for the support surface (principle of the simulation to simulate localisation data), and for the target surface (evaluation of the deviation of the localised tool, to generate a command). In the simulation, the tool is localised with a couple of coordinates for example, which permit to compute (in a function) the orthogonal projection on the axis, and a corresponding curvilinear abscissa.

\section{Equations of the movements needed by the principle of the simulation}

The equations below determine the trajectory of the tractor. They are available on the tangent plane of the support surface where the tractor is. The tractor is considered as a 4 wheel vehicle [4]. Figure 7 shows schematically the parameters used by the equations.

$\mathrm{d} \sigma=\left(\frac{\text { speedait }}{1-\rho(\mathrm{M}) \lambda}\right)$ cosyaw
$\mathrm{d} s=(\mathrm{d} \sigma) \cos$ gradient $(\mathrm{M})$
$\mathrm{d} \lambda=($ speeddt $)$ sinyaw
dyaw $=$ speeddt $\left(\frac{\tan \alpha}{\mathrm{e}}-\frac{\rho(\mathrm{M}) \operatorname{cosyaw}}{1-\rho(\mathrm{M}) \lambda}\right)$
dtan $\alpha=$ d $\alpha\left(1+\tan ^{2} \alpha\right)$

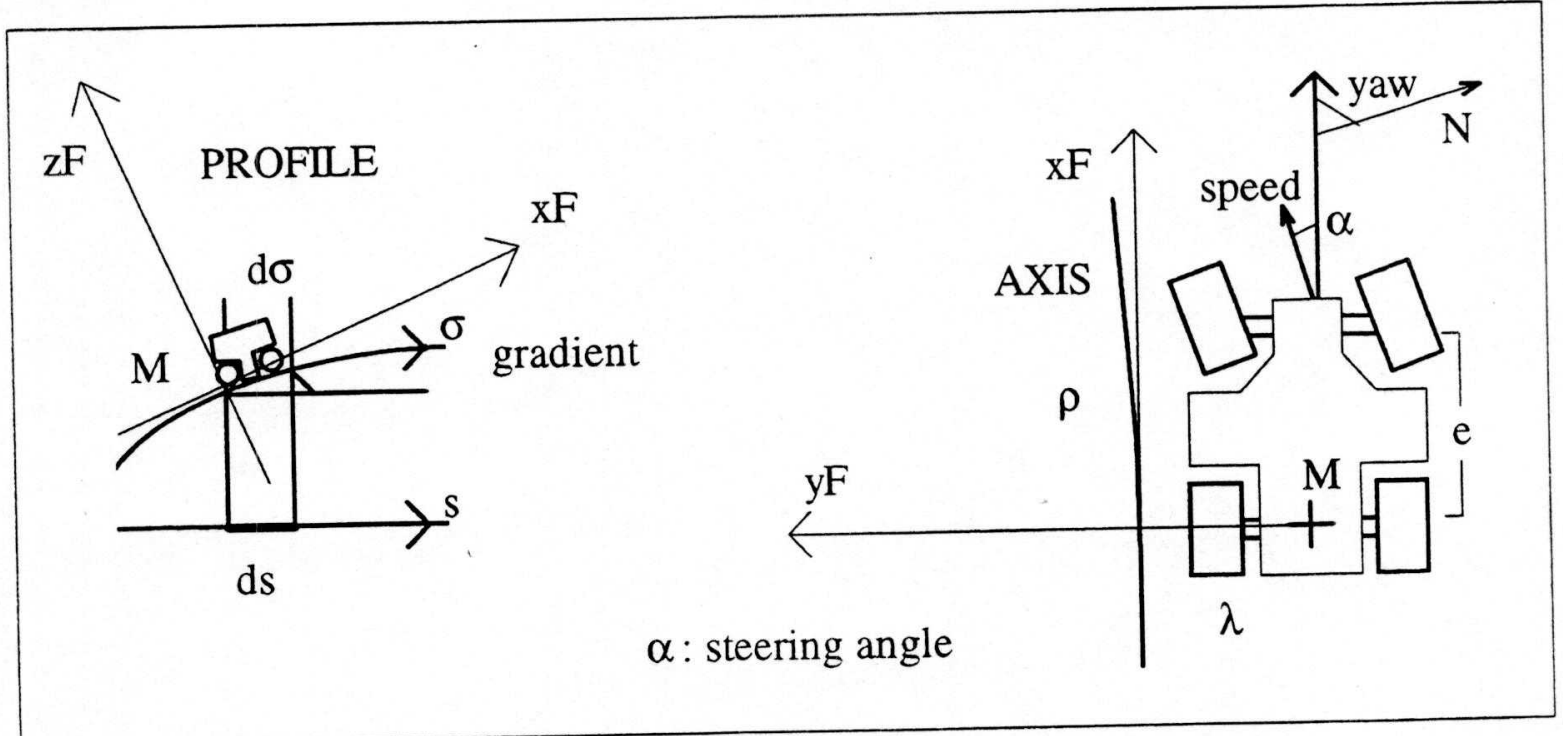

Figure $7:$ the tractor model

The model equations are available on the local (in M) tangent plane to which is associated a moving reference called Frenet: xFrenet $(\sigma)$ is the axis of the road in M, yFrenet $(\lambda)$ is supported by the cross-fall in $M$, and $z$ Frenet $(z)$ is orthogonal to $X$ and $Y$. The abscissa $\sigma$ increase along $x$ Frenet, $\lambda$ is positive when the vehicle is on the left side, negative when it is on the right side. 
Other equations determine the movement of the floating screed [5].

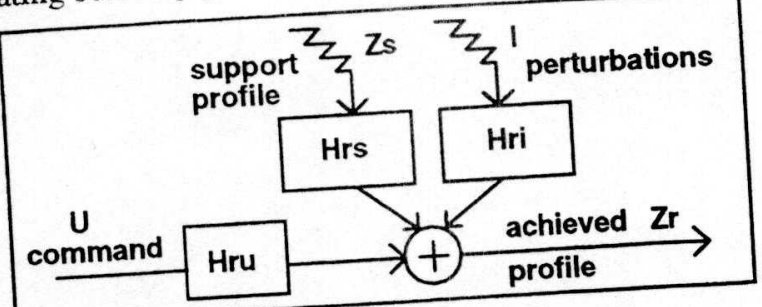

Figure 8 : the floating screed model

The transfer functions represent :

- Hru : the influence of each actuator on the height of the corresponding edge of the floating screed,

- Hrs : the influence of the support surface on the floating screed,

- Hri : the influence of variations of the parameters supposed to be constant, but sensitive to noise (speed, physical parameters of the material, vibrators, tampers...).

Hrs incorporate the transfer function of the tractor: Hch.

These transfer equations were numerically validated on an experimental paver at the LCPC. Results are used here in the software throughout a SIMULINK system.
In this system, the model was duplicated for the two actuators of the paver which are independent: the support surface presented before is indeed different near one actuator from the other.

\section{The closed-loop controller}

Automatic control of the system is possible with the simulator. Concerning the closed-loop controller for the steering command, it was natural to process a state system composed of yaw and lateral position from the The controller is based on the state feedback.

The system is observed computing the $\mathrm{X}$ and $\mathrm{Y}$ position of the floating screed wich are the simulated measurements.

Concerning the closed-loop controller for the actuators, classical transfer functions were used. Knowing that the floating screed is a non-minimal phase system, a phase-advancing integral and proportional controller has been designed.

The system is observed through the simulated $\mathrm{Z}$ position.

Figure 9 shows the complete SIMULINK diagram with all the blocks interacting between each other.

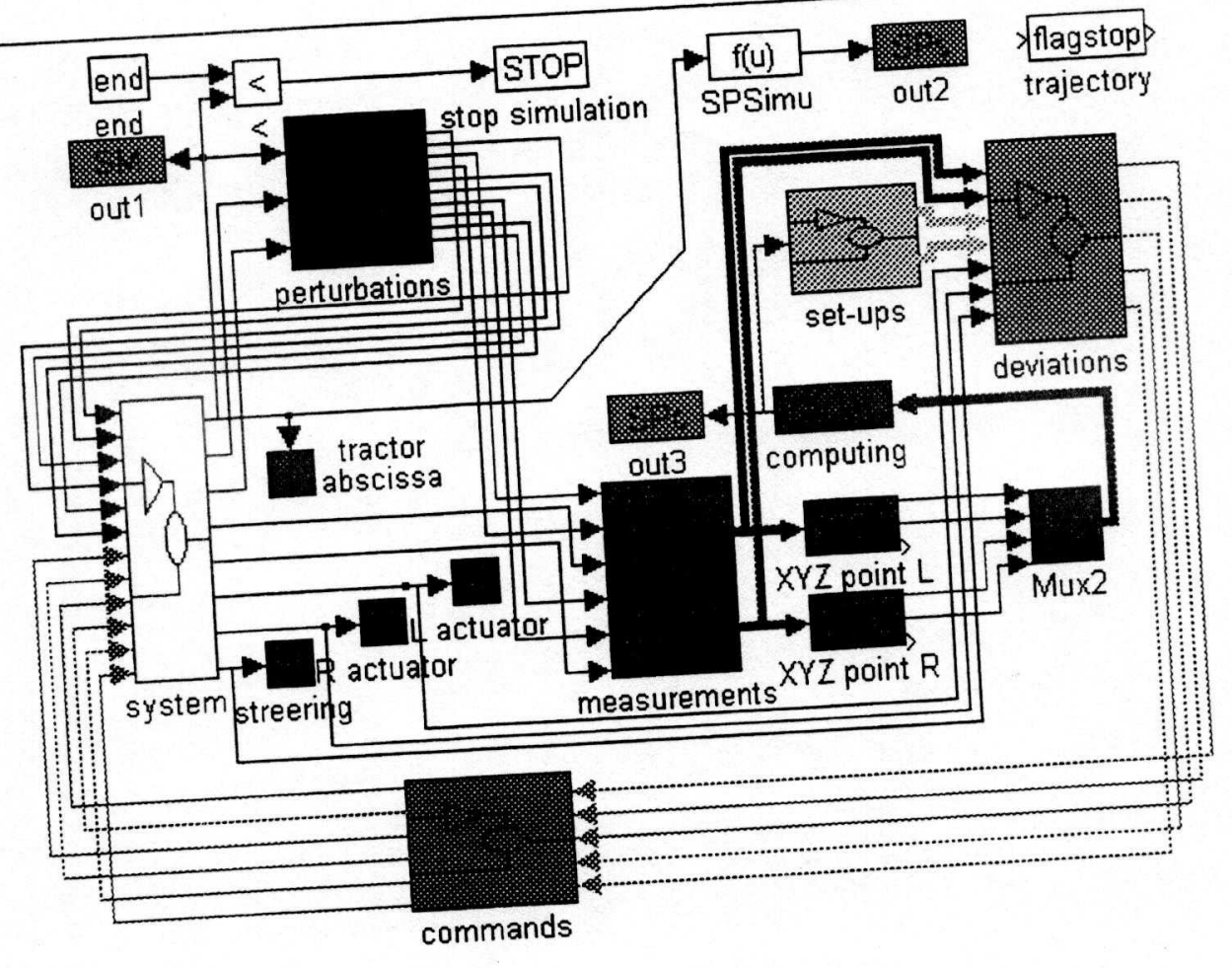

Figure 9 : the main sub-systems blocks of the simulator, and the links between them 


\section{The graphical operator's interface}

Like in many machines, the graphical operator's interface should be very clear and understandable. It is displayed to the operator as soon as a project has been chosen, with one layer in particular to be achieved, and a machine to work with.

Figure 10 shows the first version of the operator's interface.

The schematic representation is focused on the paver, and not on a general view of the works site. The left-hand side figure represents the position of the tractor relatively to the axis ( $\mathrm{X}$ and $\mathrm{Y}$ Frenet's reference). It permits the operator to drive the tractor, thanks to the steering command. The scale is about $1 \mathrm{~cm}$ for a meter. The right-hand side figure represents the position of the floating screed (working unit) relatively to the standard cross-section. The vertical scale $(\mathrm{Z})$ must be sensitive enough to view drifts of about $1 \mathrm{~cm}$, so that the operator can apply corrections using the actuators command.

The commands are sliders which are activated by clicking with the mouse : for the steering command, the slider is directly linked to the steering angle $(\alpha)$. For the floating screed, two sliders are linked to the lengths of the corresponding actuators. Both steering angle and actuators lengths are limited (saturation). In the model of the paver, no transfer function has been designed for steering and the actuators themselves, whose dynamics is supposed to be much faster than the paving process itself.

The sliders are coupled with alarms blocks, which are set when deviations exceed values of tolerance level for the layer concerned.

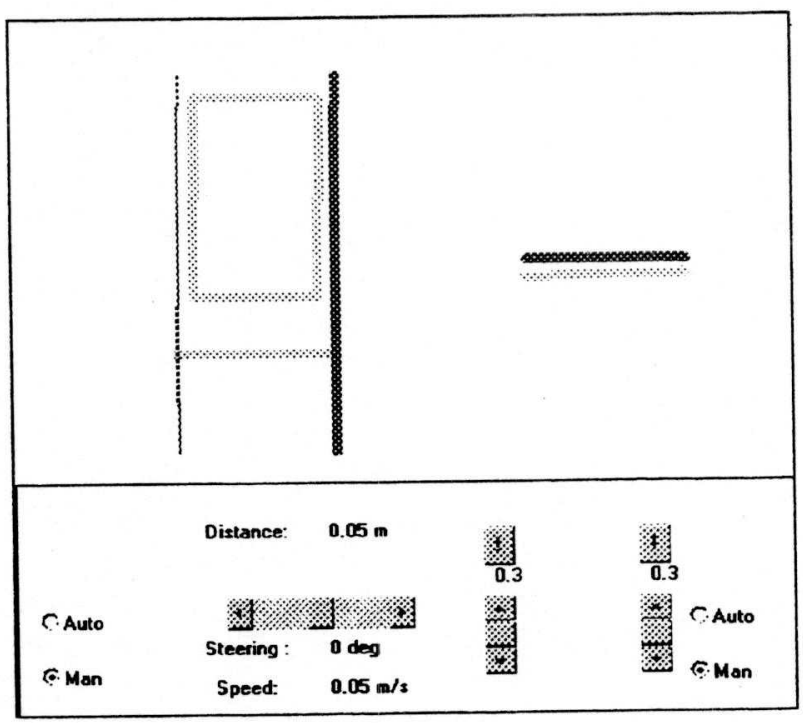

Figure $10:$ a first version of an operating aiding system
When the automatic command is clicked, the sliders display the output of the close-loop controller. Automatic and manual control can be switched on and off at any moment.

Finally, the graphical operator's interface of the simulator was designed with regard to the possible development of an operational (and not simulated) mockup of a system which could be used in an application inspired by the concept of computer integrated road construction. The graphical operator's interface can be considered as a first version of an operating aiding screen.

\section{Some results of simulation for the paver}

As a first demonstration, the efficiency of the automatic control system can be shown.

Figure 11 shows the tractor trajectory obtained when the automatic control of the steering angle is performed.

Figures 12 and 13 concern the elevation control of the screed.

On figure 12 , no automatic control is performed.We can see that the support surface is duplicated with the same defects when paving, except some smoothing of the defects under 3 meters long (length of the arms). The screed follows the support surface and not the target surface.

When automatic control is performed (figure 13) the surface achieved by the paver fits perfectly the target surface.

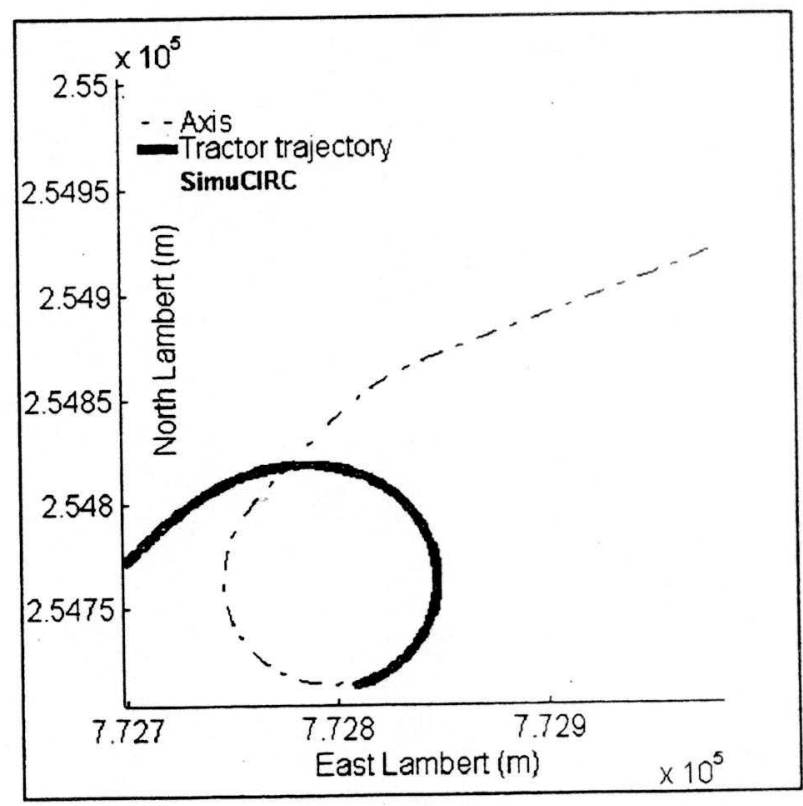

Figure 11 : plane trajectory

(automatic steering control ON) 


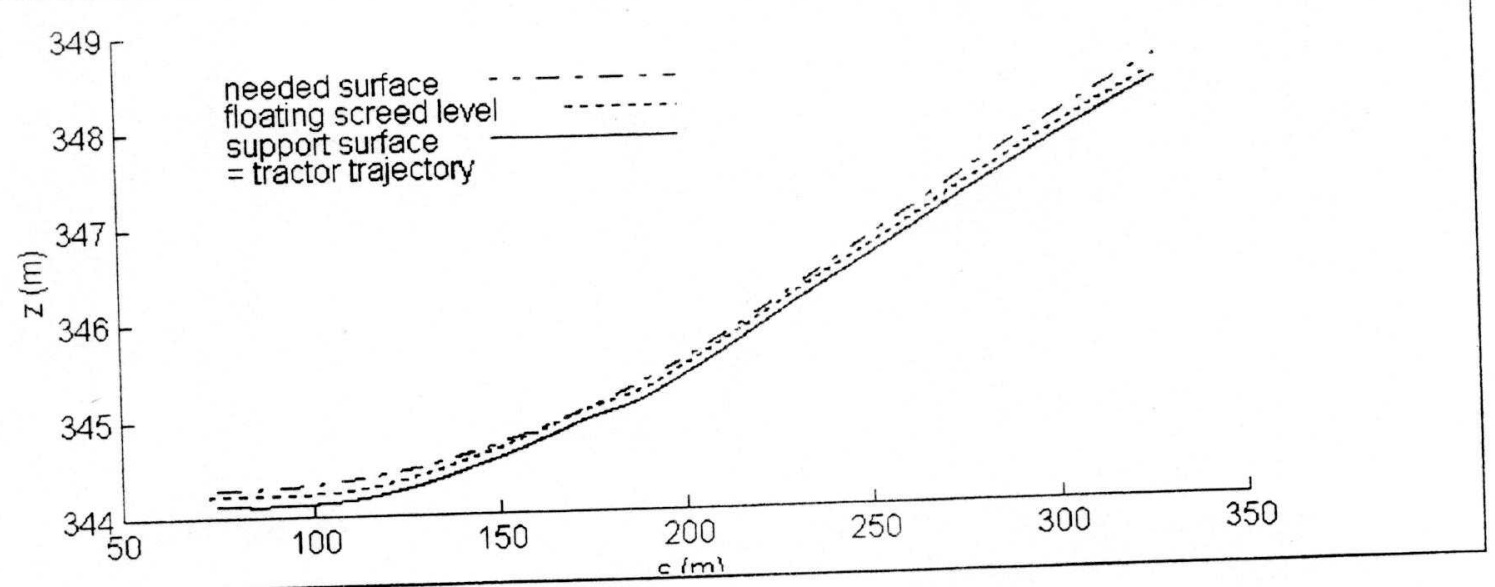

Figure 12 : profile trajectory (automatic level control OFF)

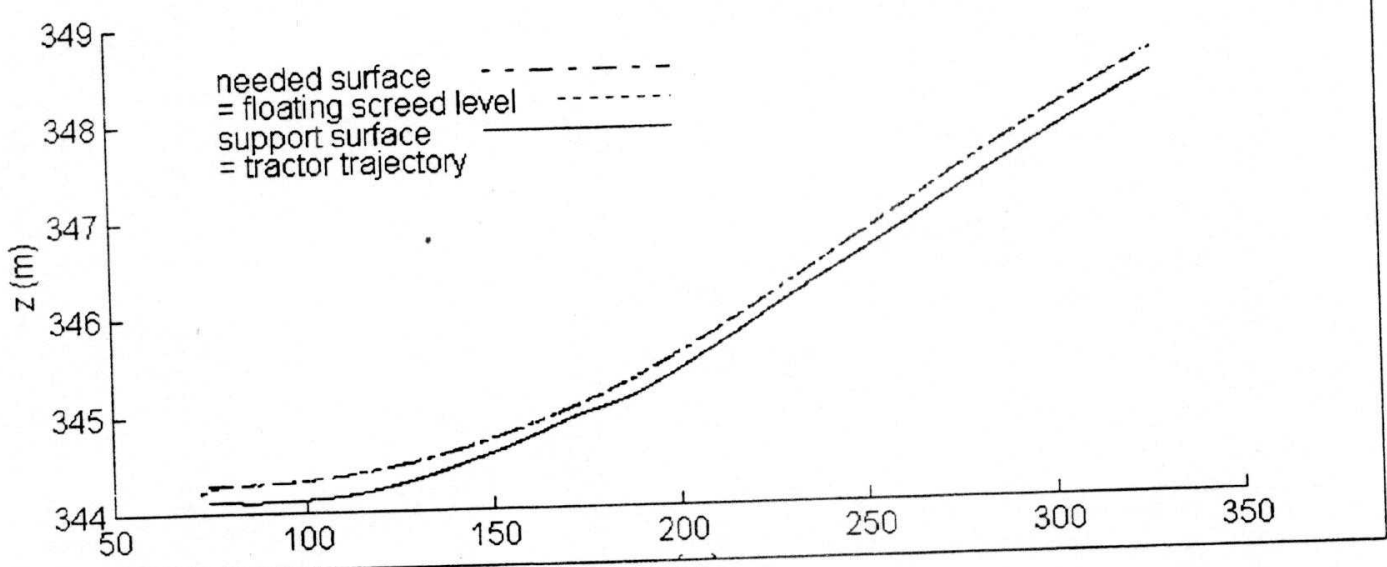

Figure 13 : profile trajectory (automatic level control ON)

\section{Conclusion}

The simulator has now been in its operational phase for about one year.

Is has already proved to be an efficient environment to support research actions in the field of mobile robotics, through common work carried out with the University of Nantes.

It is presently supporting a new action dealing with the optimal control in elevation of the screed of the paver, using GPS measurements as positioning data.

As a demonstration tool, towards road construction companies, it has also proved to be convincing in several sotuations.

In a near future, it is intended to be used by the third class of customers, that is to say the industrial integration companies, in the frame of a European project.

\section{References}

[1] Beliveau Y.J.

3-D positioning for construction surveying and automation Construction Congress II., ASCE, Boston, Mass, pp. 656-661, April 1991

[2] Peyret F., Philippe H

Towards Computer Integrated Road Construction ISARC 9, Tokyo, pp. 859-868, June 1992

[3] Ulrich $A$.

Automatic levelling in road construction ISARC 8, Stuttgart, pp. 467-478, June 1991

[4] Samson C.

Commande de véhicules non holonomes pour le suivi de trajectoires et la stabilisation vers une posture désirée Automatique pour les véhicules terrestres, Amiens, 1993

[5] Gourdon J.L., Peyret F.

Modelling and controlling the road finishing process

ISARC 8, Stuttgart, pp. 479-488, June 1991 